\title{
THE X-RAY VIEW OF THE WOLF-RAYET STARS
}

\author{
A.M.T. POLLOCK \\ Computer \& Scientific Co. Ltd, 34 Westwood Road, Sheffield S11 7EY, England
}

\begin{abstract}
Ever since the earliest work, X-ray observations have been central in the chequered development of the study of interacting high-velocity winds in binaries. This review assesses how confidently the observations of Wolf-Rayet stars may be interpreted as the result of colliding winds, drawing attention to the wide range of $\mathrm{X}$-ray luminosities even among the relatively bright binaries. Although the data do not generally conform to the simplest binary models, some suggestions are made to reconcile theory and observation.
\end{abstract}

Key words: stars: Wolf-Rayet - X-rays - colliding winds

\section{The potential and prehistory of Wolf-Rayet binary X-ray observations}

$\mathrm{X}$-ray transitions involve the innermost atomic electrons and thus, in principle, provide a means of assessing chemical abundances, via both thermalemission-line and photoelectric-absorption-edge spectra between 0.1 and 10 $\mathrm{keV}$, that is not compromised by the difficulties at longer wavelengths concerning ionisation balance. They are thus of special relevance to the study of the Wolf-Rayet stars that are almost universally accepted to be chemically evolved. At the same time, the ideas of Prilutskii \& Usov (1976) and Cherepashchuk (1976) that the collision of the two supersonic winds in a WR binary system should cause a bright, extensive X-ray temperature shock to form between the stars was so beautifully simple and easily understood that serious attempts were even made with the first generation of X-ray satellites to observe them by Cooke et al.(1978); in the event without success. The unexpected discovery with the Einstein Observatory that single hot stars are $\mathrm{X}$-ray sources irrespective of their binary status needlessly diverted attention for several years. Claims that the observed levels of X-ray emission of binaries were typical of the single stars and that there was thus no evidence for colliding-wind emission ignored the fact that the observed luminosities were orders of magnitude lower than the model predictions which, though crude, were not in obvious error. Even now, the most sophisticated numerical hydrodynamic models conform to the basic scheme proposed in 1976 of a shock near the stagnation point of the flow subject to highly variable binary-phase-related absorption when viewed through the different regions of the outflowing gas. Nevertheless, although it is now established observationally that colliding-wind X-ray emission does occur, the models still have some way to go to account for all the measurements to hand, primitive as they are. 
The basic assumption is incontrovertible that the winds of WR and $O$ stars are optically thick to photoelectric absorption such that any base $\mathrm{X}$ ray corona would effectively be invisible to an outside observer. This is most obviously demonstrated by the neutron-star X-ray binaries like 4U1700-37 (e.g., Haberl et al.1989), where the wind-accreting compact object acts as a very bright test particle near the base of the wind of an otherwise unexceptional $\mathbf{O}$ star emitting X-rays that suffer many optical depths of absorption. As discussed below, WR140 provides a similar, if less spectacular, demonstration for the WR stars.

Berghöfer \& Schmidt (1994) recently made the discovery of fundamental importance that the instrinsic X-ray emission of single $\mathrm{O}$ stars is constant. In a sample of many stars they were able to detect only one episode of variability, amounting to $15 \%$, in one star. Whatever the mechanism reponsible for the generation of X-rays in single-star winds - and the case is far from proven for relatively many small shocks driven by radiative instabilities (micro-shocks, perhaps, as opposed to colliding-wind macro shocks) - this lack of variability is also likely to apply to the instrinsic emission of single WR stars.

\section{The view before the launch of ROSAT}

After early reports, notably by Seward \& Chlebowski (1982), Moffat et al.(1982), Sanders et al.(1985) and White \& Long (1986), it was not until the uniform analysis by Pollock (1987b) of all 48 WR stars observed with the Einstein Observatory that a clear picture started to emerge and a second step was taken towards the rehabilitation of Prilutskii \& Usov's and Cherepashchuk's colliding winds. Pollock found among those 48 stars, whose $\mathrm{X}$-ray luminosities covered a wide range, that the WN stars were 4 times brighter on average than the WC stars; that the brightest X-ray stars were also among the then recently-discovered non-thermal radio stars; and that the massive binaries were brighter than the single stars, whose emission was weak enough to escape detection in many cases. He suggested that colliding winds could be the explanation for some, or even most, of this despite the inability of the theoretical models to reproduce luminosities and temperatures as low as those observed.

In addition to the statistical approach, several individual stars were worthy of note. Seward and Chlebowski (1982) remarked on the exceptional and constant brightness of WR25 (HD 93162) that still remains a mystery; a first attempt by Moffat et al. (1982) on WR139 (V444 Cyg) to observe the binaryrelated brightness variations expected of colliding winds showed a definite but unexpectedly small modulation; and of the handful of stars with more than one observation, WR6 (HD 50986) showed long-term variability, falling in luminosity by a factor of 3 over an 18 month period, implying that the 
$\mathrm{X}$-rays too arise in a system more exotic than a single-star wind.

The most excitement was generated by WR140, which has come to play a central role for colliding-wind observers and theorists after the discovery of its very eccentric, 7.94-yr binary orbit through Williams's patience waiting for the repeatable giant IR outburst. Pollock (1987a) had earlier found with EXOSAT that WR140 was a very bright X-ray source and the subsequent multiwavelength campaign during the 1985 periastron passage described by Williams et al. (1990) made some interesting discoveries. Although the $\mathrm{X}$-ray coverage was meagre, it was quite clear that the $\mathrm{X}$-ray absorption increased near periastron before decreasing rapidly afterwards as the $\mathrm{O}$ and $\mathrm{WR}$ stars retreated from each other. Undoubtedly, the X-ray source and the non-thermal radio source both follow an orbit with respect to the WR star similar in geometry to the 0 -star and it was natural to identify them with the region of the collision of the two winds between the stars. The increased absorption also demonstrated the thickness to X-rays of WR star material and tempted Williams et al. to estimate its CNO abundance as these elements are particularly effective absorbers. They calculated a fractional abundance $n_{C} \sim 0.06$ under the assumption that the emission was a smooth power-law continuum. It is clear now, as it should have been then, that the form of the underlying continuum is quantitatively very important for the derivation of chemical abundances as features occur in both emission and absorption and the energy resolution of a typical X-ray instrument is not adequate to distinguish them. A recent $A S C A$ spectrum described below has confirmed earlier reports of an Fe emission line at $6.8 \mathrm{keV}$, leading to the expectation of other line features at lower energies that is bound to effect the abundance modelling of the observed spectra.

\section{Recent results from ROSAT and $A S C A$}

\subsection{ThE ROSAT SURVEY AND OTHER DEDICATED OBSERVATIONS}

Pollock, Haberl \& Corcoran (these proceedings) report on preliminary results of the $0.2<E(\mathrm{keV})<2.4$ survey of the WR stars giving almost complete coverage of the galactic stars. It was achieved as part of the wider ROSAT PSPC all-sky survey. Results for the optically brightest stars are shown in Table I, where ' $l$ ' and ' $h$ ' denote low and high mass systems respectively. Examples are present here of the trends that apply to the entire sample and conform to expectations based on the 48 Einstein stars, particularly the brightness of the binaries in comparison to the single stars excepting the infamous and unexplained WR25 - and of the WN in comparison to the WC stars. 
TABLE I

The optically brightest stars, arranged in decreasing $v$ magnitude, in Pollock, Haberl \& Corcoran's preliminary ROSAT survey (these proceedings)

\begin{tabular}{rllcrll}
\hline WR & spectral type & type & $\begin{array}{c}v \\
(\mathrm{mag})\end{array}$ & \multicolumn{2}{c}{$L_{X}$} \\
& & & $\left(10^{32}\right.$ ergs s $\left.^{-1}\right)$ \\
\hline 11 & WC8+O9I & SB2 & 1.74 & 0.50 & \pm & 0.02 \\
48 & WC6+O9.5I & hSB1 & 5.69 & 14.03 & \pm & 0.53 \\
22 & WN7+abs & hSB1 & 6.44 & 0.00 & \pm & 0.25 \\
24 & WN7+abs & single & 6.49 & 0.21 & \pm & 0.15 \\
78 & WN7 & single & 6.61 & 0.00 & \pm & 0.23 \\
133 & WN4.5+O9.5Ib & SB2 & 6.70 & 11.79 & \pm & 1.71 \\
6 & WN5 & lSB1 & 6.94 & 4.35 & \pm & 0.14 \\
79 & WC7+O5-8 & SB2 & 6.95 & 0.59 & \pm & 0.11 \\
140 & WC7+O4-5 & SB2 & 7.07 & 116.00 & \pm & 1.05 \\
90 & WC7 & single & 7.45 & 0.00 & \pm & 0.31 \\
136 & WN6 & single & 7.65 & 0.19 & \pm & 0.08 \\
40 & WN8 & single & 7.85 & 0.00 & \pm & 0.55 \\
139 & WN5+O6 & SB2 & 8.10 & 4.30 & \pm & 0.55 \\
138 & WN6+O9 & SB2 & 8.10 & 3.76 & \pm & 1.00 \\
137 & WC7+OB & SB2 & 8.15 & 0.61 & \pm & 0.10 \\
25 & WN7+abs & single & 8.17 & 1031.39 & \pm & 57.88 \\
111 & WC5 & single & 8.23 & 0.12 & \pm & 0.03 \\
134 & WN6 & lSB1: & 8.23 & 0.46 & \pm & 0.22 \\
42 & WC7+O7V & SB2 & 8.25 & 0.00 & \pm & 0.14 \\
\hline
\end{tabular}

\subsubsection{The massive binary WR stars}

Before the ROSAT survey, WR133 (HD 190918) was the optically brightest massive WR binary that had not been observed before at $\mathrm{X}$-ray frequencies. Its discovery as a luminous $\mathrm{X}$-ray source is strong support for the notion that the WR binaries are often much brighter than single stars. In addition to the more well-known examples, other notable binary X-ray sources are WR97 (HDE 320102), whose rather unregarded Einstein detection is confirmed in the ROSAT survey, WR93 (HD 157504), and WR43 (HD 97950), the multiple stellar system at the nucleus of the giant $\mathrm{H}$ II region NGC 3603 . These are welcome results for colliding-wind enthusiasts. Less comfortable is the fact that, in common with some of the single stars, a significant number of WR binaries are weak X-ray sources, particulary among the WC stars like WR42 and WR70. A successful theory must be able to account, for example, for both WR140 and WR70. According to colliding-wind theory, all other things being equal, the $\mathrm{X}$-ray luminosity scales as the inverse of the binary 


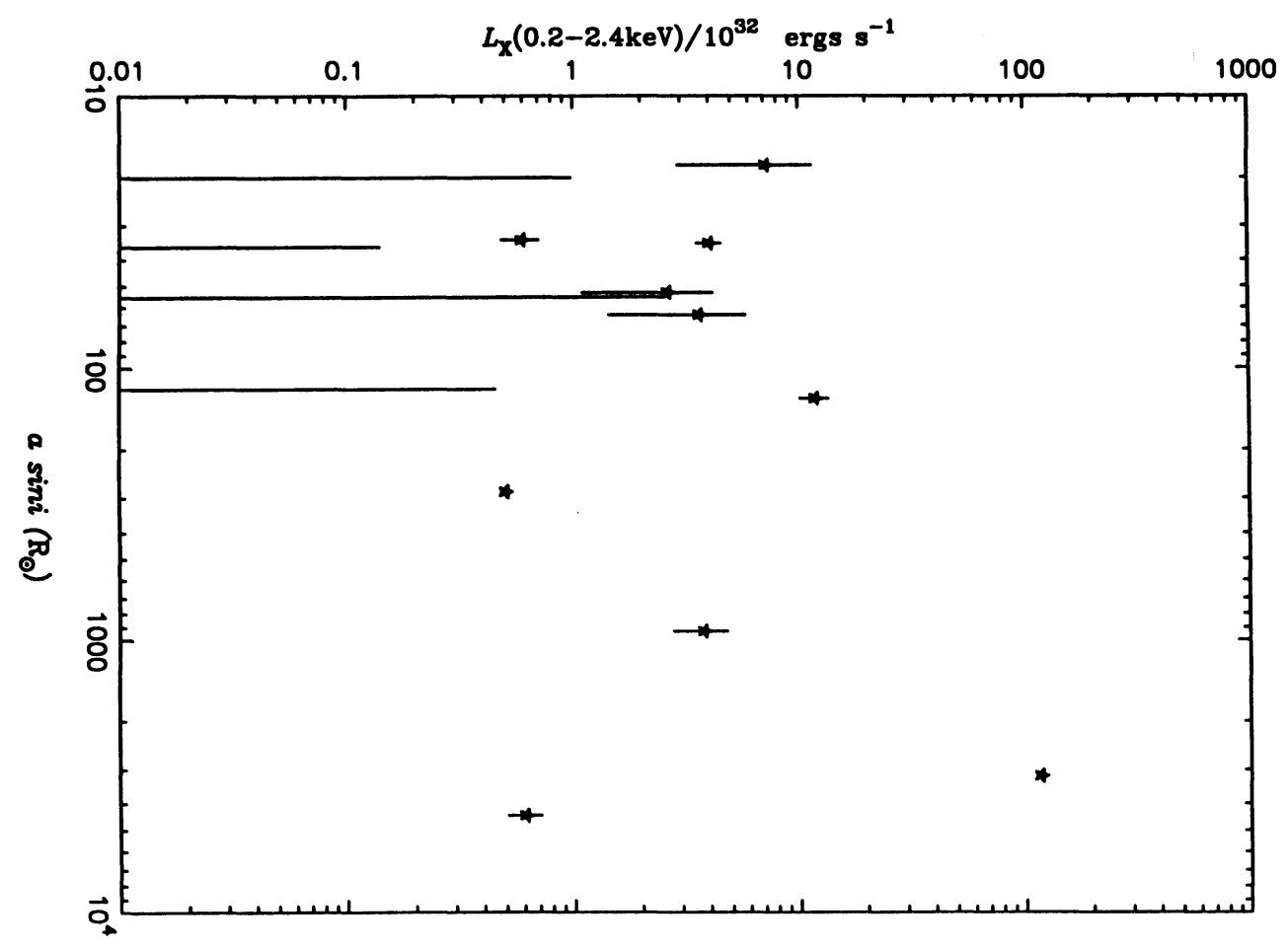

Fig. 1. ROSAT measurements of the X-ray luminosities of WR binaries plotted against separation of the component stars. The inverse linear correlation predicted by colliding-wind theory does not appear to hold.

separation (Luo et al.1990). The observed relationship is plotted in Fig. 1 and bears no resemblance to the theoretical prediction.

\subsubsection{The (apparently) single $W N$ and $W C$ stars}

The single stars are generally faint with only upper limits available for some of even the optically brightest stars surveyed. However, long pointed observations of WR111 (WC5) by Pollock et al. (these proceedings) and of several WN stars by Wessolowski et al. (these proceedings) show that X-ray luminosities of a few $\times 10^{31} \mathrm{ergs} \mathrm{s}^{-1}$ are typical of single stars although there are both brighter and fainter stars. Wessolowski et al.'s table makes especially interesting reading. The $9 \mathrm{WN}$ stars they observed showed luminosities that covered a range of 2 orders of magnitude from almost a day looking in vain at WR16 (HD 86161) $\left(L_{X} \leq 10^{31}\right.$ ergs s$\left.^{-1}\right)$ to the strong detection of WR1 (HD 4004) $\left(L_{X} \sim 10^{33} \mathrm{ergs} \mathrm{s}^{-1}\right)$ that was confirmed independently in the survey. The question then arises of whether a high luminosity shows a previously unidentified binary, for it is certain that there are some of these hiding 
among the optically fainter stars that understandably have been observed less intensively at all wavelengths.

\subsection{ROSAT OBSERVATIONS OF WR BINARIES}

Following Moffat et al.(1982), several groups have made measurements at intervals round an orbit in order to search for the binary-related modulation so confidently predicted by the models. The most successful were Willis et al.(these proceedings) with $\gamma$ Velorum, who detected a sharp increase in the X-ray flux as the $\mathrm{O}$-star passes in front of the WR star that was perfectly repeated in the later cycle they observed. The increase is confined to energies above $1 \mathrm{keV}$ and is very probably an absorbed view of colliding winds. Although Willis et al.argue that the low level emission is identified with the single WC star in the system this would seem not to be entirely the case given the significant variability observed there too. Corcoran et al.(these proceedings) showed measurements of V444 Cyg which strengthen Moffat et al.'s (1982) Einstein work and confirm the modest variability observed round the orbit at a level, however, much lower than predicted and without the expected accompanying changes in the spectrum. The least successful were Pollock, Haberl and Corcoran (these proceedings) whose six measurements of $\theta$ Muscae at regular intervals around the 18-day binary orbit showed no signs of variability, as might have been expected from what is almost certainly a triple system.

\subsection{ASCA AND ROSAT OBSERVATIONS OF WR140}

The most recent periastron passage of WR140 took place in 1993. Pollock et al.(these proceedings) reported some ROSAT measurements made before periastron which varied with binary separation in reasonable agreement with the colliding-wind models. It was after periastron that potentially the most interesting measurements were made. The ASCA satellite is providing by far the highest quality X-ray spectra ever achieved of WR stars, giving the prospect of excellent chemical abundance measurements. Skinner et $a l$. (these proceedings) showed a heavily absorbed $0.5-8 \mathrm{keV}$ X-ray spectrum of WR140 taken about 3 months after periastron and made some non-solar spectral models that fitted the data reasonably well. However, Skinner et al. 's models did not work so well on Pollock et al. 's (these proceedings) spectrum taken a few months later when the absorption had decreased. ASCA data are good enough that the thermal plasma codes like that of Raymond and Smith that have served the X-ray community so well for many years are now themselves under test. 


\section{Suggestions for a unified colliding-wind scheme}

It has been a puzzling feature of the X-ray phenomenology of the WR stars that the brightest star, WR140, is a much more distant - and presumably more weakly interacting - binary than the fainter but closer, more strongly interacting systems like V444 Cyg. It has been suggested, for example, that in close systems the winds collide at velocities much lower than the terminal value because, perhaps, of errors in the velocity law. There is, nevertheless, a simple possible explanation of the current data in which, broadly speaking, the three distinct levels of observed X-ray luminosity are associated in decreasing order of brightness with optically-thin binaries, optically-thick binaries, and single stars. The variable-absorption 2900-d-period WR140 demonstrates just how far apart have to be the WR and $O$ star binary components for a clear view of the central stagnation-point X-ray source between the stars. It is here that the colliding-wind models work best. In the 80-day period $\gamma$ Velorum Willis et al.'s ROSAT observation shows that a heavily absorbed hard X-ray source just makes an appearence during a small phase interval as the $\mathrm{O}$ star passes in front of the WR star. Closer binaries would usually be expected to be comprehensively smothered by both WR and $O$ star absorption. What, then, is the explanation of few-day period systems like V444 Cyg that, at the same time, are much fainter than both WR140 and theoretical expectations but perhaps an order of magnitude brighter than the single WR stars? Could it be this: Prilutskii \& Usov and Cherepashchuk and the other models were right after all and the instrinsic luminosity is high but, because of absorption, all we see is an electron scattered component? X-rays from the stagnation point passing too close to either of the component stars cannot escape the system but photons travelling roughly along the interaction cone will suffer the smallest absorption and a fraction should be directed towards the observer by electron scattering, whose optical depth is likely to be substantial; a scattered component is required to explain the residual flux seen during the eclipse of the neutron star by the O-star primary in 4U1700-37 (e.g., Haberl \& White 1990). This would account for both the low observed luminosities and the lack of heavy phaserelated modulation. The shock would cross the line-of-sight in favourable circumstances of inclination and geometry giving two narrow $\mathrm{X}$-ray maxima at the appropriate binary phases but the coverage achieved so far has been so extremely poor - even the relatively well-studied V444 Cyg has only been covered for a few percent of an orbit - that it could easily have escaped detection. Good phase coverage is essential for significant progress in the study of colliding winds but will require a major investment of observation time that allocation committees have been reluctant to approve. WR140 and $\gamma$ Velorum show that it should be worth it. 


\section{Acknowledgements}

Grateful thanks go to the IAU for the award of a grant.

\section{References}

Berghöfer, T., Schmidt, J.H.M.M. 1994, $A \& A$ submitted

Cherepashchuk, A.M. 1976, Sov. Astron. (Letters) 2, 138

Cooke, B.A., Fabian, A.C., Pringle, J.E. 1978, Nature 273, 645

Haberl, F., White, N.E., Kallman, T.R. 1989, ApJ 343, 409

Haberl, F. White, N.E. 1990, ApJ 361, 225

Luo, D., McCray, R., MacLow, M.-M. 1990, ApJ 362, 267

Moffat, A.F.J., Firmani, C., McLean, I.S., Seggewiss, W. 1982, in: C.W.H. de Loore \& A.J. Willis (eds.), Wolf-Rayet Stars : Observations, Physics, Evolution, Proc. IAU Symp. No. 99 (Dordrecht: Reidel), 577

Pollock, A.M.T. 1987a, $A \& A$ 171, 135

Pollock, A.M.T. 1987b, ApJ 320, 283

Prilutskii, O.F., Usov, V.V. 1976, Sov. Astron. 20, 2

Sanders, W.T., Cassinelli, J.P., Myers, R.V., van der Hucht, K.A. 1985, ApJ 288, 756

Seward, F.D., Chlebowski, T. 1982, ApJ 256, 530

White, R.L., Long, K.S. 1986, $A p J$ 310, 832

Williams, P.M., van der Hucht, K.A., Pollock, A.M.T., Florkowski, D.R., van der Woerd, H., Wamsteker, W.M. 1990, MNRAS 243, 662

\section{DISCUSSION:}

Cherepashchuk: Thank you very much for excellent results. As far as I understand, the differences between X-ray luminosities of WR stars obtained by Einstein and ROSAT observations are due to differencies in X-ray ranges. Am I right?

Pollock: For the highest count rate stars the Einstein and ROSAT luminosities compare like this:

$\begin{array}{lcc} & \text { Einstein } & \text { ROSAT } \\ & \mathrm{L}_{\mathrm{x}}(0.2-4.0 \mathrm{keV}) & \mathrm{L}_{\mathrm{x}}(0.2-2.4 \mathrm{keV}) \\ 10^{32} \mathrm{erg} \mathrm{s}^{-1} & 10^{32} \mathrm{erg} \mathrm{s}^{-1} \\ \gamma \text { Vel } & 1.1 \pm 0.2 & 0.50 \pm 0.02 \\ \theta \text { Muscae } & 20 . \pm 3 . & 14 . \pm 1 . \\ \text { EZ CMa } & 3-9 \mathrm{v} & 2-5 \mathrm{v}\end{array}$

There should be a systematic difference L(Einstein)/L(ROSAT) $<1.3$ 
Meurs: What did you assume for the X-ray spectra in order to quote all those X-ray luminosities?

Pollock: The X-ray spectrum assumed was the best fit spectrum to WR $25=\mathrm{HD} 93162$ derived by Mike Corcoron. It was a two temperature thermal model with both interstellar and circumstellar absorption.

Schulte-Ladbeck: If anyone would ever give us an X-ray polarimeter, we might be able to look at the collision region then, according to your model. Could you please tell us more about WR6? Pollock: If the X-ray emission is essentially thermal as expected from wind collision I wouldn't expect to see any polarisation in a direct view. If, however, scattering is important the tyupe of binary-related effects seen in optical polarisation may be generated. Incidentally, I see no prospects of such observations being technically feasible for many years.

Willis: I can answer that, since the ROSAT observations of HD50896 were mainly made in my Guest Observer programmes. The ROSAT PSPC (0.1--2.5 keV) observations of HD50896 weere secured in our Guest Observer programmed - 10 observations - and show about a factor of 2 variation with epoch. Within the 9 observations obtained over 6 consecutive days, the variability is smaller, less than about $30 \%$, and shows no evidence for any 'phase-dependence' in the 3.7 day "period", but some evidence for a rouch 1-day timescale of variation, which is similar to the recurrence timescale of about 1 day we have found in the UV P-Cygni profile variability in several of our IUE monitoring campaigns on HD50896. The preliminary results of our ROSAT observations of HD 50896 are published in the proceedings of the Quebec, Isle de Coudes Meeting on "Instability and Variability of Hot Star Winds", (Willis et al. 1994, Kluwer Acad. Pub., in press), and more details will be found in an A\&A publication which is currently in preparation.

Koenigsberger: Is the X-ray spectrum of the wind-wind collision region expected to be much softer than the X-ray spectrum of an accreting collapsed object? Is there a difference in the "hardness" ratio of the X-ray spectra of WR binaries vs. "single" WRs?

Pollock: The X-ray spectra of the accreting neutron star, in the 4U 1700-37 for example, is very hard and seen up to $20-30 \mathrm{keV}$ with a characteristic temperature perhaps 10 times that expected for colliding winds. Regarding your second question, the data are mainly too poor to tell although some work on individual objects could still be done on the binaries, while for single stars there's no hope at the moment.

Hill: Among the WC + O star binaries you note that there is a lack of correlation between X-ray luminosity and orbital separation. Many of the $\mathrm{O}$ stars lacked a luminosity class so this may not be much of a point, but the two lowest values of $\mathrm{Lx}$ were for systems with a main sequence $\mathrm{O}$ star.

Pollock: You are right to point out that the luminosity is bound to depend on the spectral type and luminosity class of the O-star companion. But I think this should be a second order effect compared with the complete lack of a $1 / \mathrm{D}$ correlation

Moffat: If the filling factor is low, as may be quite possible (this meeting), X-rays will be attenuated, but they will also be able to "leak out". Could this not explain the two problems you noted for binaries; namely, that Lx is low and rises little?

Pollock: I don't think so because I wouldn't expect the filling factor to be low enough for X-rays to avoid absorption all the way out of the wind. If X-rays did leak out wouldn't you expect these objects to be brighter than expected rather than the reverse? 